\title{
ADJOINTS OF IDEALS IN REGULAR LOCAL RINGS
}

\author{
JOSEPH LIPMAN \\ with an Appendix by \\ Steven Dale CutKosky
}

Introduction. Several existing results labeled "Briançon-Skoda theorem" concern an ideal $I$ in a regular local ring $R$. Of these, the weakest states that if $I$ is generated by $\ell$ elements then $\overline{I^{n+\ell-1}} \subset I^{n}(n>0)$, where "-" stands for "integral closure." In this paper, we associate to $I$ an integrally closed ideal $\widetilde{I} \supset \bar{I}$, the adjoint of $I$, and indicate how $\widetilde{I}$ can be used in place of $\bar{I}$ to improve such results. At first, in Theorem (1.4.1), this just involves a recycling of methods from [LS]. (Even that is not without benefit, see Cor. (1.4.4)). But there's more. It's not hard to show that there is an $n_{0}$ such that $\widetilde{I^{n+1}}=I \widetilde{I^{n}}$ for all $n \geq n_{0}$. The basic conjecture (1.6)-which, as we'll see, quickly implies a number of recently proved Briançon-Skoda-type theorems - says that $n_{0}$ can be taken to be the dimension of the closed fibre in the blowup of $I$, i.e., to be $\ell(I)-1$ where $\ell(I)$ is the analytic spread of $I$. This conjecture does hold when $R$ is essentially of finite type over a field of characteristic zero, or when $\operatorname{dim} R=2$.

Section 2 deals with a conjecture, related to Grauert-Riemenschneider vanishing, about certain cohomology groups being zero. Suppose there exists a proper birational map $f: Y \rightarrow \operatorname{Spec}(R)$ such that $I \mathcal{O}_{Y}$ is invertible and $Y$ is nonsingular, i.e., locally regular. (The existence of such a $Y$ in all characteristics is not yet certain, but it is needed in the vanishing conjecture.) Let $\omega_{Y}$ be a dualizing sheaf for $f$, chosen to be canonical in the sense that its restriction to the open set $U$ where $f$ is an isomorphism is $\mathcal{O}_{U}$. While the definition (1.1) of $\widetilde{I}$ uses neither $Y$ nor any duality theory, Proposition (1.3.1) states that $H^{0}\left(Y, I \omega_{Y}\right)=\widetilde{I}$; and the vanishing conjecture states that $H^{i}\left(Y, I \omega_{Y}\right)=0$ for all $i>0$.

1991 Mathematics Subject Classification. 13H05, 13C99, 14F17.

Partially supported by the National Security Agency. 
The point is that this vanishing conjecture implies conjecture (1.6). In fact it is only via Cutkosky's transcendental proof of vanishing (see Appendix) that we know conjecture (1.6) holds in characteristic zero.

Section 3 elaborates on the two-dimensional case, where the vanishing conjecture is known to hold, see Remark $(2.2 .1)$ (b). In this case a geometrically motivated treatment of the adjoint of a simple complete ideal $I$ was given in [L4], where close connections with the multiplicity sequence and the conductor ideal of the local ring $\mathfrak{o}$ of the "generic curve through $I$ " were brought out. (Roughly speaking, $\mathfrak{o}$ is the local ring at the generic point of the exceptional divisor - a $\mathbb{P}^{1}$ - on the blowup $Y_{0}$ of any 2-generated reduction $I_{0}$ of $I$.) Propositions (3.1.2) and (3.2.2) below explore such connections for an arbitrary integrally closed $I$ primary for the maximal ideal. $\widetilde{I}$ is shown to be the unique integrally closed ideal whose order is $r-1$ at any infinitely near point where $I$ has order $r>1$, and 0 elsewhere. If $Y$ is the normalization of $Y_{0}$, then $I \omega_{Y}$ is just the conductor $\mathfrak{C}:=\mathcal{O}_{Y_{0}}: \mathcal{O}_{Y}$ (so $\mathfrak{C}$ is independent of the choice of $I_{0}$ ), and it is generated by its global sections, i.e., by $\widetilde{I}$. We also find in Proposition (3.3) that $\widetilde{I}=I_{0}: I$.

Again for an integrally closed $m$-primary ideal $I$ in a two-dimensional regular local ring $(R, m)$, Huneke and Swanson have a remarkable characterization of $\widetilde{I}$ as being the second Fitting ideal $\mathfrak{F}_{2}(I)$; and more generally (since $\left.I=\mathfrak{F}_{1}(I)\right)$, for all $n>0, \widetilde{\mathfrak{F}_{n}(I)}=\mathfrak{F}_{n+1}(I)$ (see $[\mathrm{HS}]$ ).

Let me mention in closing that though the material in [L4] dates back to 1966 , the results in this paper all came out of an effort to analyze the Briançon-Skoda theorem (3.3) in [AH1].

1. Adjoints and Briançon-Skoda theorems. Let $R$ be a regular noetherian domain with fraction field $K$, let $v$ be a valuation of $K$ whose valuation $\operatorname{ring} R_{v}$ (with maximal ideal $m_{v}$ ) contains $R$, and let $h$ be the height of the prime ideal $p:=m_{v} \cap R$. We say that $v$ is a prime divisor of $R$ if $R_{v} / m_{v}$ has transcendence degree $h-1$ over its subfield $R_{p} / p R_{p}$. It is equivalent that $R_{v}$ be essentially of finite type over $R$, or that $v$ be a Rees valuation of some $R$-ideal $I$, i.e., that $R_{v}$ be $R$-isomorphic to the local ring of a point on the normalized blowup $\bar{Y}_{I}:=\operatorname{Proj}\left(\oplus_{n>0} \overline{I^{n}}\right)$, where $\overline{I^{n}}$ is the integral closure of $I^{n}$. Such a $v$ is a discrete rank-one valuation. (See [A, p. 300, Thm.1(4) and p. 336, Prop. 3]. Note also that $R$, being universally catenary, satisfies the "dimension formula" [EGA III, (5.6.4) and (5.6.1) (c)]; and that $\bar{Y}_{I}$ is of finite type over $R$ [Re, p. 27, Thm.1.5].) 
Definition (1.1). The adjoint of an $R$-ideal $I$ is the ideal

$$
\widetilde{I}:=\bigcap_{v}\left\{r \in K \mid v(r) \geq v(I)-v\left(J_{R_{v} / R}\right)\right\}
$$

where the intersection is taken over all prime divisors $v$ of $R$, and where for any essentially finite-type $R$-subalgebra $S$ of $K$, the Jacobian ideal $J_{S / R}$ is the 0-th Fitting ideal of the $S$-module of Kähler differentials $\Omega_{S / R}^{1}$.

Remarks (1.2). (a) $\widetilde{I} \subset R$ because $R$ is the intersection of its localizations at height one primes, and each such localization is the valuation ring of a $v$ for which $v\left(J_{R_{v} / R}\right)=0$. Hence

$$
\widetilde{I}=\bigcap_{v}\left\{r \in R \mid v(r) \geq v(I)-v\left(J_{R_{v} / R}\right)\right\}
$$

where the intersection is taken over all prime divisors $v$ such that $v(I)>0$.

(b) Being an intersection of valuation ideals, $\widetilde{I}$ is integrally closed; and if $\bar{I}$ is the integral closure of $I$ then

$$
I \subset \bar{I} \subset \widetilde{I}=\widetilde{\bar{I}} \text {. }
$$

In fact, for any height $h$ prime ideal $P$ such that $R / P$ is regular,

$$
\bar{I}: P^{h-1} \subset \widetilde{I} .
$$

To see this we need only prove, for any prime divisor $v$, the inequality $v\left(J_{R_{v} / R}\right) \geq v\left(P^{h-1}\right)$, for which purpose we can localize at $p:=m_{v} \cap R$, reducing to where $R$ is local; but then $R_{v} \supset R^{\prime}:=R\left[x_{2} / x_{1}, \ldots, x_{h} / x_{1}\right]$ for some $\left(x_{1}, x_{2}, \ldots, x_{h}\right)$ generating $P$, and

$$
J_{R_{v} / R}=J_{R_{v} / R^{\prime}} J_{R^{\prime} / R}=P^{h-1} J_{R_{v} / R^{\prime}}
$$

(see [LS, p. 201, (1.1) and top of p. 202]), whence the conclusion.

(c) For any $x \in R$, we have $\widetilde{x I}=x \widetilde{I}$. In particular, $\widetilde{x R}=x R$.

(d) For any two $R$-ideals $I, J$, we have $\widetilde{J I}: I=\widetilde{J}$. In particular,

$$
\widetilde{I^{n+1}}: I=\widetilde{I^{n}} \quad(n \geq 0) \text {. }
$$

(1.3). Given any finite-type birational map $f: Y \rightarrow \operatorname{Spec}(R)$, we mayand will-identify $\mathcal{O}_{Y}$ with a subsheaf of the constant sheaf $K$ on $Y$, so that the stalks $\mathcal{O}_{Y, y}(y \in Y)$ are all $R$-subalgebras of $K$. If $g: Z \rightarrow \operatorname{Spec}(R)$ is another such map which factors via $f$, then $g$ is uniquely determined by $Z$ and $Y$, and we say that $Z$ dominates $Y$. The relative Jacobian $\mathcal{J}_{f}$ (or, less precisely, $\mathcal{J}_{Y}$ ) is the coherent $\mathcal{O}_{Y}$-module whose sections over any affine open $\operatorname{Spec}(A) \subset Y$ are given by $H^{0}\left(\operatorname{Spec}(A), \mathcal{J}_{Y}\right)=J_{A / R}$.

We set

$$
\omega_{Y}:=\mathcal{O}_{Y}: \mathcal{J}_{Y} \cong \mathcal{H o m}_{Y}\left(\mathcal{J}_{Y}, \mathcal{O}_{Y}\right) .
$$

If $Y$ is normal, $\omega_{Y}$ is a canonical dualizing sheaf for $f$ [LS, p. 206, (2.3)]. 
For any proper birational $f: Y \rightarrow \operatorname{Spec}(R)$ with $Y$ normal and $I \mathcal{O}_{Y}$ invertible, we set

$$
\widetilde{I}_{Y}:=H^{0}\left(Y, I \omega_{Y}\right),
$$

the ideal obtained by restricting the intersection in Definition (1.1) to those $v$ such that $R_{v}$ is $\mathcal{O}_{Y, y}$ for some $y \in Y$. So $\widetilde{I} \subset \widetilde{I}_{Y}$, and $\widetilde{I}_{Y}$ is a "decreasing" function of $Y$ in the sense that for any proper birational $g: Z \rightarrow Y$ with $Z$ normal, we have $\widetilde{I}_{Z} \subset \widetilde{I}_{Y}$. For any prime divisor $w$, $R_{w}$ is the local ring of a point on some such $Z$; so the intersection of all $\widetilde{I}_{Z}$ is just $\widetilde{I}$.

Proposition (1.3.1). For any $Y$ as above and having pseudo-rational singularities (for example, $Y$ regular), $\widetilde{I}_{Y}=\widetilde{I}$. If such a $Y$ exists then for any multiplicative system $M$ in $R, \widetilde{I R_{M}}=\widetilde{I} R_{M}$.

Proof. The pseudo-rationality assumption forces $g_{*}\left(I \omega_{Z}\right)=I \omega_{Y}$ for all $g: Z \rightarrow Y$ as above (by [LT, p. 107, Corollary], and since $I \mathcal{O}_{Y}$ is invertible), whence

$$
\widetilde{I}_{Z}=H^{0}\left(Z, I \omega_{Z}\right)=H^{0}\left(Y, g_{*} I \omega_{Z}\right)=\widetilde{I}_{Y}
$$

and $\widetilde{I}_{Y}=\cap_{Z} \widetilde{I}_{Z}=\widetilde{I}$. The rest follows from compatibility of $H^{0}\left(Y, I \omega_{Y}\right)$ with localization on $R$.

Remarks (1.3.2). (a) That a regular $Y$ with $I \mathcal{O}_{Y}$ invertible always exists has been announced by Spivakovsky, but details have not appeared at the time of this writing. For the equicharacteristic zero case, see $[\mathrm{H}]$.

(b) In dimension 2, every normal $Y$ birationally dominating $\operatorname{Spec}(R)$ has pseudo-rational singularities, [L1, p. 212, §9], [LT, p. 103, Example (a)]. So in Proposition (1.3.1), we could take $Y$ to be the normalized blowup of $I$.

(c) An example where $Y$ in (1.3.1) can be taken to be the blowup of $I$ is given by $I=\left(x_{1}, \ldots, x_{r}, y\right) R$, where $\left(x_{1}, \ldots, x_{r}\right)$ is a regular sequence such that $R /\left(x_{1}, \ldots, x_{r}\right) R$ is still regular [LS, p. 219, Proposition, (ii)]. Here $\widetilde{I^{n}}=\widetilde{I^{n}}{ }_{Y}(n \geq 0)$ is easily calculated: for, if $L$ is any $R$-ideal generated by a regular sequence of length $\delta$ and such that all the powers of $L$ are integrally closed, then the blowup $X$ of $L$ is normal and $\mathcal{J}_{X}=L^{\delta-1} \mathcal{O}_{X}$, [LS, top of p. 202]. It follows that

$$
\begin{aligned}
\widetilde{L^{n}} & =R & & (n<\delta) \\
& =L^{n-\delta+1} & & (n \geq \delta) .
\end{aligned}
$$

For $L=I$ we have $\delta=r$ or $r+1$ according as $y$ is or is not in $\left(x_{1}, \ldots, x_{r}\right) R$. 
(d) If $R$ is local with maximal ideal $m$, and we set, for any $R$-ideal $I$,

$$
r=r(I):=\operatorname{dim}_{R / m}\left(\left(I+m^{2}\right) / m^{2}\right),
$$

then $I$ contains an ideal $I_{0}:=\left(x_{1}, \ldots, x_{r}, y\right) R$ as in (c), where we can take $\delta=r+1$ unless $R / I$ is regular, in which case $\delta=r$. If $I \neq 0$ then $\delta \geq 1$, and for $n \geq \delta$,

$$
\widetilde{I^{n}}: I^{n-\delta+1}=\widetilde{I^{\delta-1}} \supset \widetilde{I_{0}^{\delta-1}}=R,
$$

(see Remark $(1.2)(\mathrm{d}))$ whence, since $\widetilde{I^{n}}$ is integrally closed,

$$
\overline{I^{n-\delta+1}} \subset \widetilde{I^{n}} .
$$

(1.4). The following is clearly related to the Briançon-Skoda theorem in [LS, p. 204, Thm. $1^{\prime \prime}{ }^{1}{ }^{1}$ (Recall from (1.2.1) the inclusion $\bar{I} \subset \widetilde{I}$, where $\bar{I}$ and $\widetilde{I}$ are the integral closure and adjoint, respectively, of the $R$-ideal $I$. See also (1.3.3).)

We say that $I$ is $\ell$-generated $(\ell \geq 0)$ if $I$ is generated by $\ell$ elements.

Theorem (1.4.1). For any $\ell$-generated ideal $I$ in a regular noetherian domain $R$ :

(i) $\widetilde{I^{n+\ell-1}} \subset I^{n}$ for all $n \gg 0$.

(ii) If the graded ring $\operatorname{gr}_{I} R:=\oplus_{n \geq 0} I^{n} / I^{n+1}$ contains a homogeneous regular element of positive degree, then (i) holds for all $n \geq 1$.

(iii) $\widetilde{I^{n+\ell}} \subset I^{n}$ for all $n \geq 0$.

Proof. If $Y_{0}:=\operatorname{Proj}\left(\oplus_{n \geq 0} I^{n}\right)$ is the blowup of $I$, and $Y$ is its normalization, then as in [LS, p. 200, Thm. 2 and proof of Corollary], $I^{\ell-1} \omega_{Y} \subset \mathcal{O}_{Y_{0}}$ (all inside the constant sheaf $K$ on $Y_{0}$ ). Hence

$$
\widetilde{I^{n+\ell-1}} \subset H^{0}\left(Y, I^{n+\ell-1} \omega_{Y}\right) \subset H^{0}\left(Y_{0}, I^{n} \mathcal{O}_{Y_{0}}\right)=\bigcup_{j \geq 0} I^{n+j}: I^{j}
$$

For $n \gg 0, H^{0}\left(Y_{0}, I^{n} \mathcal{O}_{Y_{0}}\right)=I^{n}$ (by e.g., [EGA III, (2.3.1)]), ${ }^{2}$ proving (i). If $\operatorname{gr}_{I} R$ has a homogeneous regular element of positive degree, then $I^{n+j}: I^{j}=I^{n}$, proving (ii).

\footnotetext{
${ }^{1}$ For that statement and the ones that follow it to be correct, the integer $\delta(I)$ therein must be replaced by $\max (\delta(I), 0)$. (When $R$ is local, $\delta(I)<0$ iff $R / I$ is regular.)

${ }^{2}$ For a more elementary proof, apply [ZS, pp. 154-155, Lemmas 4 and 5] to the ideal $\mathfrak{B}:=(0)$ in $\operatorname{gr}_{I} R$ to find an integer $q$ such that for any $n$ and any $x \in H^{0}\left(Y_{0}, I^{n} \mathcal{O}_{Y_{0}}\right) \backslash I^{n}$, $x \notin I^{q}$ (because the leading form of $x$ annihilates all homogeneous elements of large degree ...) Such an $x$ must lie in $\overline{I^{n}}$. But there exists $p$ such that $\overline{I^{p+1}}=I \overline{I^{p}}$, whence $\overline{I^{p+q}} \subset I^{q}$, and therefore $n<p+q$.
} 
In (i), the restriction of $n$ to sufficiently large values is annoying, and may well be unnecessary (see Conjecture (1.6) below). If so, then (iii) - and the following ungainly argument-would be superfluous.

The polynomial ring $R[t]$ is still regular. An immediate consequence of the following Lemma is that for any $R$-ideal $L, \widetilde{L} R[t] \subset \widetilde{L R[t}]$. (The adjoints are taken in $R$ and $R[t]$ respectively.) With $I^{\prime}:=(I, t) R[t]$, we have that $\operatorname{gr}_{I^{\prime}} R[t] \cong\left(\operatorname{gr}_{I^{\prime}} R\right)[t]$ has a regular element (namely $t$ ) of degree 1 , and we can apply (ii) to get $\widetilde{I^{\prime n+\ell}} \subset I^{\prime n}$ for all $n \geq 0$; and since $\widetilde{I^{n+\ell}} R[t] \subset\left(I^{n+\ell} R[t]\right)^{\sim} \subset \widetilde{I^{\prime n+\ell}}$ and $I^{\prime n} \cap R=I^{n}$, therefore (iii) results.

Lemma (1.4.2). Let $w$ be a prime divisor of the polynomial ring $R[t]$ and let $v$ be the restriction of $w$ to $K$, the fraction field of $R$. Then $v$ is a prime divisor of $R$, and for any $R$-ideal $L$,

$$
v(L)-v\left(J_{R_{v} / R}\right) \geq w(L)-w\left(J_{R_{w} / R[t]}\right) .
$$

Proof. Let $\left(R_{w}, m_{w}\right)$ and $\left(R_{v}, m_{v}\right)$ be the (discrete) valuation rings of $w$ and $v$ respectively. Set $q:=m_{w} \cap R_{v}[t]$. There are two cases to consider.

(1) $q=m_{v} R_{v}[t]$. Then the localization $R_{v}[t]_{q}$ is a discrete valuation ring contained in, and hence equal to, $R_{w}$. Thus $R_{w} / m_{w}=\left(R_{v} / m_{v}\right)(t)$ has transcendence degree (t.d.) 1 over $R_{v} / m_{v}$.

(2) $q \supsetneq m_{v} R_{v}[t]$, whence $q$ is maximal, and $R_{v}[t] / q$ is algebraic over $R_{v} / m_{v}$. Since $R_{w}$ is essentially of finite type over $R[t]$, hence over $R_{v}[t]$, therefore $w$ is a prime divisor of $R_{v}[t]$; and so $R_{w} / m_{w}$ has t.d. 1 over $R_{v}[t] / q$. Thus, again, $R_{w} / m_{w}$ has t.d. 1 over $R_{v} / m_{v}$.

Now set $p^{\prime}:=m_{w} \cap R[t]$ and $p:=p^{\prime} \cap R=m_{v} \cap R$, so that $R_{w} / m_{w}$ has t.d. height $\left(p^{\prime}\right)-1$ over $R[t] / p^{\prime}$, and by the preceding, the t.d. of $R_{v} / m_{v}$ over $R / p$ is height $\left(p^{\prime}\right)-2+$ the t.d. of $R[t] / p^{\prime}$ over $R / p$. It follows then from [ZS, p. 323, Prop. 1A] that $R_{v} / m_{v}$ has t.d. height $(p)-1$ over $R / p$, and so $v$ is indeed a prime divisor of $R$.

The last assertion follows from the relation

$$
J_{R_{w} / R[t]}=J_{R_{w} / R_{v}[t]} J_{R_{v}[t] / R[t]}=J_{R_{w} / R_{v}[t]} J_{R_{v} / R}
$$

(See [LS, p. 201, (1.1)] for the first equality.)

Suppose now that $R$ is local, with maximal ideal $m$. For an $R$-ideal $I$, the analytic spread $\ell(I)$ is the Krull dimension of the ring $\oplus_{n \geq 0} I^{n} / m I^{n}$. When $R / m$ is infinite then $I$ has an $\ell(I)$-generated reduction $\bar{I}_{0} \subset I$, i.e., $I_{0} I^{n}=I^{n+1}$ for some $n \geq 0$.

Corollary (1.4.3). For $R$ local, assertions (i) and (iii) in Theorem (1.4.1) hold with $\ell$ the analytic spread of $I$. And if $I$ has an $\ell$-generated reduction $I_{0}$ such that $g_{I_{0}} R$ contains a homogeneous regular element of positive degree, then (i) holds for all $n \geq 0$.

Proof. By arguing as in the proof of (1.4.1)(iii), with $R[t]$ replaced by its localization $S:=R[t]_{m R[t]}$, and $I^{\prime}:=I S$, we reduce to the case where $R / m$ is infinite. Then we can apply (1.4.1) to an $\ell$-generated reduction $I_{0}$, noting that for any valuation $v$ such that $R_{v}$ contains $R$ we have $v\left(I_{0}\right)=v(I)$, whence $\widetilde{I_{0}^{p}}=\widetilde{I^{p}}$ for all $p \geq 0$. 
The following statement was conjectured by Huneke.

Corollary (1.4.4). If $(R, m)$ is a d-dimensional regular local ring and $I$ is an $m$-primary ideal, then for all $n \geq 1$,

$$
\overline{I^{n+d-1}}: m^{d-1} \subset I^{n} \text {. }
$$

Proof. Replacing $(R, I)$ by $\left(S:=R[t]_{m R[t]}, I S\right)$ if necessary, we may assume that $R / m$ is infinite. Then $I$ has a $d$-generated reduction $I_{0}$ such that $\operatorname{gr}_{I_{0}} R$ is a polynomial ring in $d$ variables over $R / I_{0}$; so Corollary (1.4.3) gives $\widetilde{I^{n+d-1}} \subset I^{n}$, and we can apply (1.2.2).

Lemma (1.5). Let $R$ be a regular noetherian domain, let $I$ be an $R$-ideal, and set $G:=\oplus_{n \geq 0} I^{n}, \widetilde{G}:=\oplus_{n \geq 0} \widetilde{I^{n}}$. Then $\widetilde{G}$ is a finitely generated graded $G$-module, and hence there is an $n_{0}$ such that

$$
\widetilde{I^{n+1}}=\widetilde{I I^{n}} \text { for all } n \geq n_{0} \text {. }
$$

Proof. $\widetilde{G}$ is a graded $G$-module because, clearly, $I^{p} \widetilde{I^{q}} \subset \widetilde{I^{p+q}}(p, q \geq 0)$. Now just note, with $Y$ the normalized blowup of $I$, that by $(1.3), \widetilde{G}$ is a submodule of $\oplus_{n \geq 0} H^{0}\left(Y, I^{n} \omega_{Y}\right)$, which is finitely generated over $G$ (see [EGA III, (3.3.2)]).

As we'll see in (2.3) below, the following refinement of Lemma (1.5) holds true when $R$ is essentially of finite type over a characteristic-zero field, or when $\operatorname{dim} R=2$. (The 2-dimensional case also results from Prop. (3.1.2), or from Prop. (4.2) of [HS]. For another example, see Remark (1.3.2)(c).)

Conjecture (1.6). Let $R$ be a regular local ring, and let $I$ be an $R$-ideal of analytic spread $\ell$. Then

$$
\widetilde{I^{n+1}}=I \widetilde{I^{n}} \text { for all } n \geq \ell-1 \text {. }
$$

We illustrate the usefulness of this conjecture (when it holds) by indicating how it implies some Briançon-Skoda-type theorems recently proved for equicharacteristic regular local rings by Aberbach and Huneke. These theorems are all of the form

$$
\overline{I^{n+\ell-1}} \subset I^{n} A \quad(n>0),
$$

where the "coefficient ideal" $A$ depends only on $I$. Under the assumption that (1.6) holds, we need only show that $\widetilde{I^{\ell-1}} \subset A$ in order to get the stronger assertion (see (1.2.2) and (1.3.3)):

$$
\widetilde{I^{n+\ell-1}}=I^{n} \widetilde{I^{\ell-1}} \subset I^{n} A \quad(n>0) .
$$


(1.6.1). In [AH2] $A$ is taken to be the sum of all ideals $A^{\prime}$ such that $I A^{\prime}=\bar{I} A^{\prime}$. By (1.6) and $(1.2)(\mathrm{b}), I \widetilde{I^{\ell-1}}=\widetilde{I^{\ell}}=\bar{I} \widetilde{I^{\ell-1}}$, so that $\widetilde{I^{\ell-1}} \subset A .^{3}$

(1.6.2). In [AH1, p. 350, Thm.3.3], $A$ is taken to be the intersection of the primary components of $I^{\ell-h}$ belonging to the minimal primes $p_{1}, \ldots, p_{e}$ of $I$, where $h:=\max _{i} h_{i}:=\max _{i}$ height $\left(p_{i}\right)$. (To check that $\ell \geq h$, just localize at each $p_{i}$.) To show that $\widetilde{I^{\ell-1}} \subset A$, localize at $p=p_{i}(1 \leq i \leq e)$, and note that

$$
\widetilde{I^{\ell-1}} R_{p} \subset \widetilde{I^{\ell-1} R_{p}} \subset I^{\ell-h_{i}} \subset I^{\ell-h},
$$

where the first inclusion is elementary, and the second is given by (1.4.1)(ii).

Moreover, if (1.6) holds, then with $I_{p}:=I R_{p}$ we have

$$
\widetilde{I_{p}^{\ell-1}}=I_{p}^{\ell-h} I_{p}^{h-h_{i}} \widetilde{I_{p}^{h_{i}-1}}=I_{p}^{\ell-h} \widetilde{I_{p}^{h-1}}
$$

and hence if $\widetilde{I^{\ell-1}} R_{p}=\widetilde{I_{p}^{\ell-1}}$ for all $p_{i}$ (see Prop. (1.3.1)), then $\widetilde{I^{\ell-1}}$ is even contained in the intersection of the primary components of $I^{\ell-h} \widetilde{I^{h-1}}$ belonging to the $p_{i}$.

(1.6.3). In [AHT, Thm. 7.6], the above-mentioned Thm. 3.3 of [AH1] is strengthened. Here the inductive description of $A$ is a bit complicated. So suffice it to say that the inclusion $\widetilde{I^{\ell-1}} \subset A$ can be established by alternately localizing at suitable associated primes of height $i$ and applying (1.6), as $i$ goes, one step at a time, from $\ell-1$ down to the height of $I$.

2. A vanishing conjecture. Again, let $I$ be an ideal in a regular local ring $(R, m)$. Throughout this section we make the following assumptionwhich is satisfied at least over varieties in characteristic zero $[\mathrm{H}$, p. 143 , Cor. 1], or whenever $\operatorname{dim} R=2$, as follows e.g., from the Hoskin-Deligne formula, see [L3, p. 223, (3.1.1)].

Assumption (2.1). There exists a map $f: Y \rightarrow \operatorname{Spec}(R)$ which factors as a sequence of blowups with nonsingular centers, such that $I \mathcal{O}_{Y}$ is invertible.

The basic conjecture (1.6) will be deduced from the following vanishing conjecture.

Vanishing Conjecture (2.2). With $I$ and $f: Y \rightarrow \operatorname{Spec}(R)$ as above,

$$
H^{i}\left(Y, I \omega_{Y}\right)=0 \quad \text { for all } i>0 \text {. }
$$

\footnotetext{
${ }^{3}$ When $\operatorname{dim} R=2$ and $I$ is a 2 -generated ideal primary for the maximal ideal, then $\widetilde{I}=A$, see Prop. (3.3) below.
} 
Remarks (2.2.1). (a) Cutkosky has a proof of the vanishing conjecture for local rings essentially of finite type over a field of characteristic zero, (Appendix, Theorem A3). He uses Kodaira vanishing, which doesn't always hold in positive characteristic; but that does not preclude the conjecture for special maps such as $f$.

(b) It was noted in (1.3) that $\omega_{Y}$ is a dualizing sheaf for $f$. By duality [L2, p. 188], the conjecture is equivalent to the vanishing of $H_{E}^{i}\left(Y,\left(I \mathcal{O}_{Y}\right)^{-1}\right)$ for all $i<\operatorname{dim} R$, where $E:=f^{-1}\{m\}$ is the closed fiber. For $d=2$, this dual assertion is proved in [L2, p. 177, Thm. 2.4].

(c) For $I=R$, the conjecture is a form of Grauert-Riemenschneider vanishing, and is readily proved by induction on the number of blowups making up the map $f$. For arbitrary $I$, the conjecture is equivalent to the vanishing of $H^{i}(Y, \mathcal{Q})$ for all $i>0$ and every invertible quotient $\mathcal{Q}$ of a finite direct sum of copies of $\omega_{Y}$ (because $I \mathcal{O}_{Y}$ is a quotient of a direct sum of copies of $\mathcal{O}_{Y} \ldots$ )

Further, if $g: Z \rightarrow \operatorname{Spec}(R)$ is the normalized blowup of $I$ and $h: Y \rightarrow Z$ is the domination map, then using the Leray spectral sequence for $f=g h$, and ampleness of $I \mathcal{O}_{Z}$, one shows that the vanishing of $R^{i} h_{*} \omega_{Y}(i>0)$ is equivalent to the vanishing of $H^{i}\left(Y, I^{n} \omega_{Y}\right)$ for all $n \gg 0$. In other words, Conjecture (2.1) is somewhat stronger than Grauert-Riemenschneider vanishing for "sandwiched singularities."

(d) Theorem (4.1) of [L6, p. 153] shows that there is an $R$-ideal $L$ such that $Y$ in (2.1) is the blowup of $L$, i.e., the Proj of the Rees ring $R[L t]$ ( $t$ an indeterminate), and such that $R[L t]$ is Cohen-Macaulay (CM). This leads to another conjecture, which can be shown to imply the vanishing one:

CM Conjecture. Let $L=I I^{\prime}$, with $L, I$, and $I^{\prime}$ integrally closed $R$-ideals, and assume that $R[L t]$ is $C M$ and normal. Then for some $e>0$, the ideal $\operatorname{IR}\left[L^{e} t\right]$ (which is divisorial) is $C M$ as an $R\left[L^{e} t\right]$-module.

(2.3). We show next that Conjecture (1.6) follows from the vanishing conjecture. ${ }^{4}$ Thus (1.6) does hold for local rings of smooth points of algebraic varieties in characteristic zero, or when $\operatorname{dim} R=2$. (See the preceding remarks (2.2.1)(a) and (b).)

We reduce to where $R / m$ is infinite by passing as usual to $S:=R[t]_{m R[t]}$. We saw in proving (1.4.1)(iii) that for any $R$-ideal $L, \widetilde{L} S \subset \widetilde{L S}$; but now we need equality, which we can get by applying Prop. (1.3.1) to $Y \otimes_{R} S$, with $Y$ as in (2.1). (I don't know a more elementary way.)

Now let $I_{0}=\left(a_{1}, \ldots, a_{\ell}\right) R$ be a reduction of $I$, so that $I_{0} \mathcal{O}_{Y}=I \mathcal{O}_{Y}$. Let $F$ be the direct sum of $\ell$ copies of $\left(I_{0} \mathcal{O}_{Y}\right)^{-1}$, and let $\sigma: F \rightarrow \mathcal{O}_{Y}$ be the $\mathcal{O}_{Y}$-homomorphism defined by the sequence $\left(a_{1}, \ldots, a_{\ell}\right)$. Then we have a

\footnotetext{
${ }^{4}$ All we'll need here is that $Y$ is regular and $I \mathcal{O}_{Y}$ is invertible.
} 
locally split Koszul complex

$$
K(F, \sigma): 0 \rightarrow \Lambda^{\ell} F \rightarrow \Lambda^{\ell-1} F \rightarrow \cdots \rightarrow \Lambda^{1} F \stackrel{\sigma}{\rightarrow} \mathcal{O}_{Y} \rightarrow 0
$$

(see [LT, p. 111]), so that $K(F, \sigma) \otimes I^{n+1} \omega_{Y}(n \geq \ell-1)$ is exact. By $(2.2)$, and with $H^{i}(-):=H^{i}(Y,-)$,

$$
H^{1}\left(I^{n-1} \omega_{Y}\right)=H^{2}\left(I^{n-2} \omega_{Y}\right)=\cdots=H^{\ell-1}\left(I^{n+1-\ell} \omega_{Y}\right)=0 .
$$

Hence, as in [LT, p. 112, Lemma (5.1)] we can conclude that

$$
H^{0}\left(I^{n+1} \omega_{Y}\right)=I H^{0}\left(I^{n} \omega_{Y}\right),
$$

i.e., by Proposition (1.3.1), $\widetilde{I^{n+1}}=I \widetilde{I^{n}}$.

3. Dimension 2. Except in Lemma $(3.2 .1),(R, m)$ will be a regular local ring of dimension 2 and $I$ will be an $m$-primary $R$-ideal. The purpose of this section is to give a number of alternative descriptions of $\widetilde{I}$.

(3.1). It is pointed out in the footnote on p. 235 of [L4] that when $I$ is a simple integrally closed ideal, the definition of the adjoint of $I$ given in [L4, p. 229] and [L5, p. 299] agrees with the one in this paper (see Proposition (1.3.1)). Let us extend this result - more specifically, the not-quitecorrectly stated Corollary (4.1) of [L4, p. 233] - to arbitrary $I$.

The point basis of $I$ is the family of integers $\left(\operatorname{ord}_{S}\left(I^{S}\right)\right)_{S \supset R}$ where $S$ runs through all two-dimensional regular local rings between $R$ and its fraction field, $\operatorname{ord}_{S}$ is the order valuation associated with the maximal ideal of $S$, and $I^{S}:=(\operatorname{gcd}(I S))^{-1} I S$, the $S$-transform of $I$. There are only finitely many $S$ for which $\operatorname{ord}_{S}\left(I^{S}\right) \neq 0$; these are called the base points of $I$ [L4, p. 225]. Two $m$-primary ideals $I^{\prime}$ and $I^{\prime \prime}$ have the same point basis iff their integral closures coincide [L3, p.209, (1.10)].

Consider a sequence of regular schemes

$$
\operatorname{Spec}(R)=: X_{0} \overleftarrow{f_{0}} X_{1} \overleftarrow{f_{1}} \cdots \overleftarrow{f_{n}} X_{n+1}=: X
$$

where $f_{i}: X_{i+1} \rightarrow X_{i}(0 \leq i \leq n)$ is obtained by blowing up a point on $X_{i}$ whose local ring $\left(S_{i}, m_{i}\right)$ is a base point of $I$, and where $I \mathcal{O}_{X}$ is invertible. Denote by $m_{i} \mathcal{O}_{X}$ the invertible $\mathcal{O}_{X}$-ideal whose stalk at $x \in X$ is $m_{i} \mathcal{O}_{X, x}$ if $\mathcal{O}_{X, x} \supset S_{i}$, and $\mathcal{O}_{X, x}$ otherwise. Then

$$
\omega_{X}^{-1}=\prod_{i=0}^{n} m_{i} \mathcal{O}_{X},
$$

see (1.2.3), or the footnote on page 235 in [L4]. With $E_{i} \cong \mathbb{P}_{S_{i} / m_{i}}^{1}$ the curve on $X$ corresponding to the $m_{i}$-adic valuation, and $\left[S_{i}: R\right]$ the degree of the field extension $\left(S_{i} / m_{i}\right) /(R / m)$, we have that the intersection 
number $\left(E_{i} \cdot E_{i}\right)$ is $-d_{i}\left[S_{i}: R\right]$ for some positive integer $d_{i}$, and $d_{i}=1 \mathrm{iff}$ $I^{S_{i}}$ generates an invertible ideal on $X_{i+1}$, i.e., iff $I^{S_{i}}=m_{i}^{d}(d>0)$, in which case $\left(I \mathcal{O}_{X} \cdot E_{i}\right)=d\left[S_{i}: R\right]$. Also, as in [L4, p. 235],

$$
\left(\omega_{X} \cdot E_{i}\right)=-\left(E_{i} \cdot E_{i}\right)-2\left[S_{i}: R\right] .
$$

It follows that $\left(I \omega_{X} \cdot E_{i}\right) \geq 0$ for all $i$, so by [L1, p. 220, Thm. (12.1)(ii)], $I \omega_{X}$ is generated by its global sections, i.e., by $\widetilde{I}$, see Prop. (1.3.1). Thus:

$$
I \omega_{X}=\widetilde{I} \mathcal{O}_{X} \quad \text { i.e., } \quad I \mathcal{O}_{X}=\widetilde{I} \prod_{i=0}^{n} m_{i} \mathcal{O}_{X} .
$$

For any $S \supset R$, we have then

$$
\operatorname{ord}_{S}(I)-\operatorname{ord}_{S}(\widetilde{I})=\sum_{S_{i} \subset S} \operatorname{ord}_{S}\left(m_{i}\right) .
$$

On the other hand, setting, for any two-dimensional regular local $T$ between $R$ and its fraction field, $r_{T}:=\operatorname{ord}_{T}\left(I^{T}\right), \widetilde{r}_{T}:=\operatorname{ord}_{T}\left(\widetilde{I}^{T}\right)$, we have

$$
\operatorname{ord}_{S}(I)-\operatorname{ord}_{S}(\widetilde{I})=\sum_{T \subset S} \operatorname{ord}_{S}\left(m_{i}\right)\left(r_{T}-\widetilde{r}_{T}\right)
$$

see [L4, p. 301, Remark (1)]. By induction on the length $g$ of the unique sequence of quadratic transforms $R:=R_{0} \subset R_{1} \subset \cdots \subset R_{g}=: S$ (see [A, p. 343, Thm.3]), we deduce that

$$
\begin{aligned}
r_{S}-\widetilde{r}_{S} & =1 & & \left(S=S_{1}, S_{2}, \ldots, S_{n}\right) \\
& =0 & & \text { otherwise. }
\end{aligned}
$$

But since $S_{1}, S_{2}, \ldots, S_{n}$ are precisely the base points of $I$, i.e., those $S$ such that $r_{S}>0$, what this amounts to is that $\widetilde{r}_{S}=\left(\max \left(0, r_{S}-1\right)\right)$. Thus:

Proposition (3.1.2). $\widetilde{I}$ is the unique integrally closed ideal whose point basis is

$$
\left(\max \left(0, \operatorname{ord}_{S}\left(I^{S}\right)-1\right)\right)_{S \supset R} .
$$

For any two-dimensional regular local $T$ between $R$ and its fraction field, the point basis of the transform $I^{T}$ is obtained from that of $I$ by restriction to those $S$ which contain $T$. Moreover, a theorem of Zariski states that $I^{T}$ is integrally closed if $I$ is (see e.g., [L5, p. 300]). We have then the following generalization of [L4, p. 231, Thm. (3.1)]:

Corollary (3.1.3). Adjoint commutes with transform: for all $T, \widetilde{I^{T}}=\widetilde{I} T$. 
(3.2). For the next result, let $I$ be any non-zero integrally closed ideal in a $d$-dimensional regular local ring $R$, such that $I$ has a reduction $I_{0}$ generated by a regular sequence $\left(a_{1}, a_{2}, \ldots, a_{d}\right)$. Let $Y_{0}$ be the blowup of $I_{0}$, let $\pi: Y \rightarrow Y_{0}$ be the normalization map, and let $\mathfrak{C}$ be the conductor of $Y$ in $Y_{0}$. Then $\mathfrak{C}$ is independent of $I_{0}$ :

Lemma (3.2.1). With the preceding notation, we have $\mathfrak{C}=I^{d-1} \omega_{Y}$.

Proof. Noting that $Y_{0} \rightarrow \operatorname{Spec}(R)$ is a local complete intersection map (see [LS, top of p. 202]), and arguing as on pp. 205-207 of loc. cit., we find that

$$
\pi_{*} \omega_{Y}=\mathcal{H o m}\left(\pi_{*} \mathcal{O}_{Y}, \omega_{Y_{0}}\right)=\mathcal{H o m}\left(\pi_{*} \mathcal{O}_{Y},\left(I_{0} \mathcal{O}_{Y_{0}}\right)^{1-d}\right),
$$

so that

$$
\pi_{*} I^{d-1} \omega_{Y}=\pi_{*} I_{0}^{d-1} \omega_{Y}=I_{0}^{d-1} \pi_{*} \omega_{Y}=\mathcal{H o m}\left(\pi_{*} \mathcal{O}_{Y}, \mathcal{O}_{Y_{0}}\right)=\pi_{*} \mathfrak{C}
$$

whence the assertion.

More can be said in the two-dimensional case.

Proposition (3.2.2). With the preceding notation, when $d=\operatorname{dim} R=2$, $\mathfrak{C}$ is generated by its global sections $H^{0}(Y, \mathfrak{C})=H^{0}\left(Y, I \omega_{Y}\right)=\widetilde{I}$, i.e., $\mathfrak{C}=\widetilde{I} \mathcal{O}_{Y}$

Proof. Choose $X$ as in (3.1), and let $g: X \rightarrow Y$ be the domination map (which exists because $I \mathcal{O}_{X}$ is invertible). As in the proof of Prop. (1.3.1), $g_{*}\left(I \omega_{X}\right)=I \omega_{Y}=\mathfrak{C}$, the last equality by Lemma (3.2.1). Also, by [L1, p. 209, Prop. (6.5)], the $\mathcal{O}_{Y}$-ideal $\widetilde{I} \mathcal{O}_{Y}$ is integrally closed. Hence, and by $(3.1 .1)$,

$$
\widetilde{I} \mathcal{O}_{Y}=g_{*}\left(\widetilde{I} \mathcal{O}_{X}\right)=g_{*}\left(I \omega_{X}\right)=I \omega_{Y}=\mathfrak{C},
$$

whence the assertion.

Here is another characterization of $\widetilde{I}$.

Proposition (3.3). Let $I$ be an m-primary integrally closed ideal in a regular local ring $R$ of dimension 2 , and let $I_{0}=(a, b) R$ be a reduction of $I$. Then with $D$ an injective hull of $R / m$, we have a duality isomorphism

$$
R / \widetilde{I} \cong \operatorname{Hom}_{R}\left(I / I_{0}, D\right)
$$

Hence the $R$-module $I / I_{0}$ depends only on $I$, and has annihilator $I_{0}: I=\widetilde{I}$. 
Proof. Let $f: Y \rightarrow \operatorname{Spec}(R)$ be the normalized blowup of $I$. Recall that $H^{1}\left(Y, \mathcal{O}_{Y}\right)=0$ [L1, p. 199, Prop. (1.2)]; and $H^{2}\left(Y, \mathcal{O}_{Y}\right)=0$ since the fibers of $f$ have dimension $<2$. (In fact the following argument applies to any two-dimensional pseudo-rational singularity.) With $\mathcal{I}:=I \mathcal{O}_{Y}=(a, b) \mathcal{O}_{Y}$, we have the exact Koszul complex

$$
0 \longrightarrow \mathcal{I}^{-1} \stackrel{-b \oplus a}{\longrightarrow} \mathcal{O}_{Y} \oplus \mathcal{O}_{Y} \stackrel{(a, b)}{\longrightarrow} \mathcal{I} \longrightarrow 0
$$

whence an exact homology sequence, with $H^{\bullet}(-):=H^{\bullet}(Y,-)$,

$R \oplus R=H^{0}\left(\mathcal{O}_{Y} \oplus \mathcal{O}_{Y}\right) \stackrel{(a, b)}{\longrightarrow} H^{0}(\mathcal{I})=I \rightarrow H^{1}\left(\mathcal{I}^{-1}\right) \rightarrow H^{1}\left(\mathcal{O}_{Y} \oplus \mathcal{O}_{Y}\right)=0$

yielding

$$
I / I_{0} \cong H^{1}\left(\mathcal{I}^{-1}\right)
$$

Since $H^{1}\left(\mathcal{O}_{Y}\right)=0$ and $H^{2}\left(O_{Y}\right)=0$, therefore

$$
H^{1}\left(\mathcal{I}^{-1}\right) \cong H^{1}\left(\mathcal{I}^{-1} / \mathcal{O}_{Y}\right)
$$

Moreover, $\mathcal{I}^{-1} / \mathcal{O}_{Y}$ vanishes on $Y \backslash E:=Y \backslash f^{-1}\{m\}$; and thus

$$
H_{E}^{1}\left(\mathcal{I}^{-1} / \mathcal{O}_{Y}\right) \cong H^{1}\left(\mathcal{I}^{-1} / \mathcal{O}_{Y}\right) \cong I / I_{0}
$$

Denoting the dualizing functor $\operatorname{Hom}_{R}(-, D)$ by $-^{\prime}$, we have, by [L2, p. 188],

$$
H_{E}^{2}\left(\mathcal{I}^{-1}\right) \cong \operatorname{Ext}^{0}\left(\mathcal{I}^{-1}, \omega_{Y}\right)^{\prime} \cong H^{0}\left(\mathcal{I} \otimes \omega_{Y}\right)^{\prime}=(\widetilde{I})^{\prime}
$$

and similarly

$$
H_{E}^{2}\left(\mathcal{O}_{Y}\right) \cong \operatorname{Ext}^{0}\left(\mathcal{O}_{Y}, \omega_{Y}\right)^{\prime} \cong H^{0}\left(\omega_{Y}\right)^{\prime}=R^{\prime}
$$

Recall from $(2.2 .1)(\mathrm{b})$ that $H_{E}^{1}\left(\mathcal{I}^{-1}\right)=0$. So there is an exact sequence

$$
0 \rightarrow H_{E}^{1}\left(\mathcal{I}^{-1} / \mathcal{O}_{Y}\right) \rightarrow H_{E}^{2}\left(\mathcal{O}_{Y}\right) \rightarrow H_{E}^{2}\left(\mathcal{I}^{-1}\right) \rightarrow 0
$$

whose dual is an exact sequence

$$
0 \rightarrow \widetilde{I} \rightarrow R \rightarrow \operatorname{Hom}_{R}\left(I / I_{0}, D\right) \rightarrow 0
$$

which gives the desired conclusion. 


\title{
Appendix: A VANISHING THEOREM FOR LOCAL RINGS
}

\author{
by Steven Dale Cutkosky ${ }^{5}$
}

The purpose of this note is to prove a variation of the Kodaira vanishing theorem which is applicable to commutative algebra. The Theorem (Theorem A3) is used above by Lipman in his proof of a very general Briançon-Skoda type theorem. In particular, this gives a proof of Lipman's Vanishing Conjecture (2.2) for rings essentially of finite type over a field of characteristic zero.

The vanishing theorem proved here is the consequence of a generalization by Ramanujam of the Kodaira vanishing theorem. The book "Vanishing theorems on projective manifolds" by Shiffman and Sommese [SS] is a good reference for vanishing theorems on projective varieties. Theorem A2, from which Theorem A3 is immediate, can be further generalized along the lines of the theorems for complex projective manifolds in [SS].

Given a field $k$, a $k$-variety $W$ and a line bundle $\mathcal{L}$ on $W$, let $L$ be a subfield of $k$ such that $W$ and $\mathcal{L}$ are defined over $L$. We will denote the corresponding $L$-variety by $W_{L}$, and the corresponding line bundle on $W_{L}$ by $\mathcal{L}_{L}$.

Theorem A1 (Ramanujam). Let $\mathcal{L}$ be a line bundle on a nonsingular complex projective variety $X$. Suppose that some positive multiple $\mathcal{L}^{\otimes m}$ is generated by global sections and the image of $X$ by a projective morphism determined by $\Gamma\left(X, \mathcal{L}^{\otimes n}\right)$ has the dimension of $X$ for large $n$. Then

$$
H^{i}\left(X, \mathcal{L}^{-1}\right)=0 \text { for } i<\operatorname{dim}(X)
$$

This is Theorem 3 of [Ra]. The proof is also given in [SS, Theorem 7.1].

Theorem A2. let $R$ be a local domain essentially of finite type over a field $k$ of characteristic zero. Suppose that $f: X \rightarrow \operatorname{Spec}(R)$ is a projective resolution of singularities, $\omega_{X}$ is a dualizing sheaf on $X$, and $\mathcal{L}$ is a line bundle on $X$ which is generated by global sections. Then $H^{i}\left(X, \mathcal{L} \otimes \omega_{X}\right)=0$ for $i>0$.

Proof. $R$ is the local ring of a point $P$ on a projective $k$-variety $V$. By resolution of singularities $[\mathrm{H}]$, there exists a nonsingular variety $Y$ and a projective morphism $a: Y \rightarrow V$ such that $a: Y_{P} \rightarrow V_{P}$ is isomorphic to $f: X \rightarrow \operatorname{Spec}(R)$. Extend $\mathcal{L}$ to a line bundle $\mathcal{L}^{\prime}$ on $Y$. Let $\mathcal{A}$ be a very ample divisor on $V$. After replacing $\mathcal{A}$ by a sufficiently high power, we

\footnotetext{
${ }^{5}$ Partially supported by NSF
} 
have that $\mathcal{A} \otimes a_{*}\left(\mathcal{L}^{\prime}\right)$ is generated by global sections on $V$, so there is a surjection

$$
\Gamma\left(V, \mathcal{A} \otimes a_{*}\left(\mathcal{L}^{\prime}\right)\right) \otimes_{k} \mathcal{O}_{V} \rightarrow \mathcal{A} \otimes a_{*}\left(\mathcal{L}^{\prime}\right)
$$

$\mathcal{L}^{\prime} \mid Y_{P}$ is generated by global sections, so that

$$
a_{*}\left(\mathcal{L}^{\prime}\right) \otimes_{\mathcal{O}_{V}} \mathcal{O}_{Y} \rightarrow \mathcal{L}^{\prime} \text { is surjective in a neighborhood of } Y_{P}
$$

By (1) and (2), and since $\Gamma\left(Y, \mathcal{A} \otimes \mathcal{L}^{\prime}\right)=\Gamma\left(V, \mathcal{A} \otimes a_{*}\left(\mathcal{L}^{\prime}\right)\right)$, therefore $\Gamma\left(Y, \mathcal{A} \otimes \mathcal{L}^{\prime}\right) \otimes_{k} \mathcal{O}_{Y} \rightarrow \mathcal{A} \otimes \mathcal{L}^{\prime}$ is surjective in a neighborhood of $Y_{P}$. Hence $\mathcal{A} \otimes \mathcal{L}^{\prime}$ is generated by global sections in a neighborhood of $a^{-1}(P)$.

By resolution of indeterminacies $[\mathrm{H}]$, there exists a projective birational morphism of nonsingular varieties $g: Y^{\prime \prime} \rightarrow Y$ obtained by blowing up a subscheme whose support is disjoint from $a^{-1}(P)$, and an exceptional divisor $E$ for $g$ such that $\mathcal{A} \otimes \mathcal{O}(-E) \otimes \mathcal{L}^{\prime}$ is generated by global sections. $\mathcal{A}^{2} \otimes \mathcal{O}(-E) \otimes \mathcal{L}^{\prime}$ is then also generated by global sections, and a nontrivial section of $\Gamma\left(Y^{\prime \prime}, \mathcal{A} \otimes \mathcal{O}(-E) \otimes \mathcal{L}^{\prime}\right)$ gives an inclusion

$$
\Gamma\left(Y^{\prime \prime}, \mathcal{A}\right) \subset \Gamma\left(Y^{\prime \prime}, \mathcal{A}^{2} \otimes \mathcal{O}(-E) \otimes \mathcal{L}^{\prime}\right)
$$

The image of the morphism from $Y^{\prime \prime}$ into $\mathbf{P}\left(\Gamma\left(Y^{\prime \prime}, \mathcal{A}^{2} \otimes \mathcal{O}(-E) \otimes \mathcal{L}^{\prime}\right)\right)$ projects onto $V$ in $\mathbf{P}\left(\Gamma\left(Y^{\prime \prime}, \mathcal{A}\right)\right)$. So after possibly replacing $\mathcal{A}$ by a high power, we may assume that the image of $Y^{\prime \prime}$ by the projective map determined by $\Gamma\left(Y^{\prime \prime}, \mathcal{A} \otimes \mathcal{O}(-E) \otimes \mathcal{L}^{\prime}\right)$ has dimension equal to the dimension of $V$. Let $\mathcal{M}=\mathcal{A} \otimes \mathcal{O}(-E) \otimes \mathcal{L}^{\prime}, b=a \circ g$. Then $b: Y_{P}^{\prime \prime} \rightarrow V_{P}$ is isomorphic to $f: X \rightarrow \operatorname{Spec}(R)$, and $\mathcal{M}_{P} \cong \mathcal{L}$.

$Y^{\prime \prime}$ and $\mathcal{M}$ are defined over a finitely generated extension field of the rationals. Let $L$ be an algebraic closure of this extension in the function field of $Y^{\prime \prime}$. Then $L$ can be embedded in the complex numbers $\mathbf{C}$, and $Y_{L}^{\prime \prime} \otimes_{L} \mathbf{C}$ is a nonsingular complex projective variety.

By Ramanujam's vanishing theorem (Theorem A1), $H^{q}\left(Y_{\mathbf{C}}^{\prime \prime}, \mathcal{M}_{\mathbf{C}}^{-1}\right)=0$ for $q<\operatorname{dim}(V)$. Since $H^{q}\left(Y_{\mathbf{C}}^{\prime \prime}, \mathcal{M}_{\mathbf{C}}^{-1}\right) \cong H^{q}\left(Y_{L}^{\prime \prime}, \mathcal{M}_{L}^{-1}\right) \otimes_{L} \mathbf{C}$ and since $H^{q}\left(Y_{k}^{\prime \prime}, \mathcal{M}_{k}^{-1}\right) \cong H^{q}\left(Y_{L}^{\prime \prime}, \mathcal{M}_{L}^{-1}\right) \otimes_{L} k$, we have that

$$
H^{q}\left(Y_{k}^{\prime \prime}, \mathcal{M}_{k}^{-1}\right)=0 \text { for } q<\operatorname{dim}(V) .
$$

By Serre duality, we have $H^{q}\left(Y^{\prime \prime}, \omega_{Y^{\prime \prime}} \otimes \mathcal{M}\right)=0$ for $q>0$. Consider the Leray spectral sequence

$$
H^{r}\left(V, R^{s} b_{*}\left(\omega_{Y^{\prime \prime}} \otimes \mathcal{M}\right) \otimes \mathcal{A}^{n}\right) \Rightarrow H^{r+s}\left(Y^{\prime \prime}, \omega_{Y^{\prime \prime}} \otimes \mathcal{M} \otimes \mathcal{A}^{n}\right) .
$$


$H^{r}\left(V, R^{s} b_{*}\left(\omega_{Y^{\prime \prime}} \otimes \mathcal{M}\right) \otimes \mathcal{A}^{n}\right)=0$ for $r>0$ and $R^{s} b_{*}\left(\omega_{Y^{\prime \prime}} \otimes \mathcal{M}\right) \otimes \mathcal{A}^{n}$ is generated by global sections for sufficiently large $n$ since $\mathcal{A}$ is ample. We then have

$$
H^{0}\left(V, R^{s} b_{*}\left(\omega_{Y^{\prime \prime}} \otimes \mathcal{M}\right) \otimes \mathcal{A}^{n}\right)=H^{s}\left(Y^{\prime \prime}, \omega_{Y^{\prime \prime}} \otimes \mathcal{M} \otimes \mathcal{A}^{n}\right)=0
$$

for $s>0$ and all sufficiently large $n$. Hence $R^{s} b_{*}\left(\omega_{Y^{\prime \prime}} \otimes \mathcal{M}\right) \otimes \mathcal{A}^{n}=0$ for $s>0$, and $H^{s}\left(X, \mathcal{L} \otimes \omega_{X}\right)=0$.

Theorem A3. Let $R$ be a local domain essentially of finite type over a field $k$ of characteristic zero. Suppose that $f: X \rightarrow \operatorname{Spec}(R)$ is a resolution of singularities, $\omega_{X}$ is a dualizing sheaf on $X$, and $I \subset R$ is an ideal such that $I \mathcal{O}_{X}$ is an invertible $\mathcal{O}_{X}$ module. Then $H^{i}\left(X, I \omega_{X}\right)=0$ for $i>0$.

Proof. The conclusion follows from Theorem A2 since $I \mathcal{O}_{X}$ is a line bundle generated by sections.

\section{References}

[A] S. S. Abhyankar, On the valuations centered in a local domain, Amer. J. Math. 78 (1956), 321-348.

[AH1] I. Aberbach, C. Huneke, An improved Briançon-Skoda theorem with applications to the Cohen-Macaulayness of Rees algebras, Math. Annalen 297 (1993), 343369.

[AH2] _ A theorem of Briançon-Skoda type for equicharacteristic regular local rings, in preparation.

[AHT $] —$ N. T. Trung, Reduction numbers, Briançon-Skoda Theorems, and the depth of Rees algebras, Compositio Math. (to appear).

[EGA] A. Grothendieck and J. Dieudonné, Éléments de Géométrie Algébrique, III, Publications Math. IHES, 11 (1961).

$[\mathrm{H}] \quad$ H. Hironaka, Resolution of singularities of an algebraic variety over a field of characteristic zero, Annals of Math. 79 (1964), 109-326.

[HS] C. Huneke and I. Swanson, The core of ideals in two-dimensional regular local rings, preprint.

[L1] J. Lipman, Rational singularities, with applications to algebraic surfaces and unique factorization, Publ. Math. IHES 36 (1969), 195-279.

[L2] , Desingularization of two-dimensional schemes, Annals of Math. 107 (1978), 151-207.

[L3] _ On complete ideals in regular local rings, Algebraic Geometry and Commutative Algebra, vol. I, in honor of Masayoshi Nagata, Kinokuniya, Tokyo, 1988, pp. 203-231.

[L4] - Adjoints and polars of simple complete ideals in two-dimensional regular local rings, Bull. Soc. Math. Belgique 45 (1993), 224-244.

[L5] _ Proximity inequalities for complete ideals in two-dimensional regular local rings, Contemporary Mathematics 159 (1994), 293-306.

[L6] Cohen-Macaulayness in graded algebras, Mathematical Research Letters 1 (1994), 149-157. 
[LS] and A. Sathaye, Jacobian ideals and a theorem of Briançon-Skoda, Michigan Math. J. 28 (1981), 199-222.

[LT] and B. Teissier, Pseudo-rational local rings and a theorem of BriançonSkoda about integral closures of ideals, Michigan Math. J. 28 (1981), 97-116.

[Ra] C.P. Ramanujam, Remarks on the Kodaira vanishing theorem, J. Indian Math. Soc. 36 (1972), 41-50.

[Re] D. Rees, A note on analytically unramified local rings, J. London Math. Soc. 36 (1961), 24-28.

[SS] B. Shiffman and A. Sommese, Vanishing theorems on complex manifolds, Progress in Mathematics 56, Birkhäuser, Boston, 1991.

[ZS] O. Zariski and P. Samuel, Commutative Algebra, vol. 2, van Nostrand, Princeton, 1960.

Dept. of M athematics, Purdue University, W. Lafayette in 47907, USA

E-mail address: lipman@math.purdue.edu

Dept. of Mathematics, Univerity of Missouri, Columbia Mo 65211 , Usa

E-mail address: dale@cutkosky.cs.missouri.edu 